\section{Phosphatases are emerging as novel druggable targets in Plasmodium}
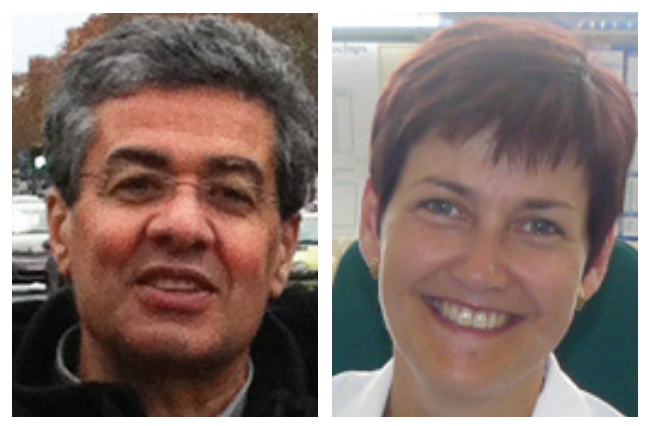

\author{
“To date only three Pf \\ phosphatases crystal \\ structures have been solved \\ and two can be considered \\ valid active phosphatases.”
}

Jamal Khalife ${ }^{*, 1} \&$ Christine Pierrot ${ }^{2}$

First draft submitted: 15 February 2016; Accepted for publication: 16 February 2016; Published online: 9 May 2016

One of the key components in the malaria control is the artemisinin-based combination therapies. However, the recent and convergent studies indicating a reduced parasite clearance rate to artemisinin are first and warning signs to continue innovation in the development of effective antimalarial drugs to counteract Plasmodium resistance. Over the last decade, the use of a wide range of approaches including biochemical, genetic and cell biology revealed that several phosphatases and their regulators are involved in essential processes in its life cycle. Targeting these enzymes by selective blocking of their activities and/or by preventing the assembly of active enzymes appears to be highly promising to offer new means in the fight against malaria.

\section{Malaria: a constant threat}

The efforts made over the last 2 decades to control malaria seem to be bearing fruit as the applied programs have led to an approximate $60 \%$ reduction in mortality rates. However, despite this trend, malaria infection is still unacceptably high ( 214 million cases in 2015) and still affects health, social and economic sectors that contribute to the wellbeing and quality of life. In addition, the control of malaria remains fragile because of: the great and sustainable efforts required to meet all recommended measures in developing countries; the increase in parasite clearance time and/or resistance to artemisinin combined therapy treatment and; the low protection provided by the latest candidate based on the RTS's malaria vaccine. With a genome encoding approximately 5600 known and predicted proteins, Plasmodium has developed a highly sophisticated program to invade two hosts and grow within diverse cell types. This multistage life cycle, combined with the dramatic and rapid amplification of parasite numbers during its sexual and asexual stages could explain at least in part the extremely challenging obstacles for the development of vaccines and potent drugs with stable efficacy. Because of this, the identification of novel means to kill the parasite and to control this disease should be a major and continual objective.

\section{KEYWORDS}

- anti-malarials $\bullet$ drugs $\bullet$ plasmodium

- phosphatases $\bullet \mathrm{PP} 1$ regulators 
"In eukaryotic cells PP1 and PP2A together are responsible for more than $90 \%$ of the protein phosphatase activity.”

\section{Phosphatases as essential as kinases in} Plasmodium

One of the central methods exploited by Plasmodium to regulate dynamic actions of its lifecycle is by engaging the phosphorylation and dephosphorylation processes of parasite proteins, linked to kinases and phosphatases, respectively. These enzymes, known to be involved in protein post-translational modifications are major pivots for protein functions and are crucial systems for therapeutic intervention against the parasite. With regard to kinases, it has become clear that they should be included in strategies for antiparasitic drug discovery. A series of recent reviews provides a comprehensive and extensive survey about kinases as rational drug targets not only in eukaryotic pathogens but also in cancer [1-4].

Similar to protein kinases, protein phosphatases could now be considered as one of the major regulatory systems exploited by Plasmodium for its survival, but this pathway has been very little explored in the past. In Plasmodium, the classification of putative and experimentally characterized protein phosphatases showed that they can be categorized as serine/threonine, tyrosine and dual phosphatases. Serine/threonine phosphatases seem to represent the largest group while protein tyrosine phosphatases are underrepresented in Plasmodium when compared with human homologs. Earlier studies on Plasmodium falciparum, devoted to the characterization of phosphatases by measuring their activities and by using natural inhibitors of Ser/Thr phosphatases, showed that protein phosphatase type 1 (PfPP1) activity is predominant when compared with protein phosphatase $2 \mathrm{~A}$ (PP2A) [5]. This was subsequently confirmed by transcriptomic analysis in which the transcript levels of PfPP1 are much higher than those detected for PP2A, particularly at $24 \mathrm{~h}$ post infection of red blood cells (PlasmoDB). It has also to be noted that okadaic acid and calyculin A, effective inhibitors of PP1 and PP2A, drastically inhibited parasite growth in vitro. Although very few phosphatases have been characterized, as producing recombinant active enzymes is still challenging in Plasmodium, studies using reverse genetic approaches reported that 16 protein phosphatases out of 30 examined, including PP1 and putative phosphatases, seem to be essential for blood stage parasites: no viable $\mathrm{KO}$ parasites could be obtained [6]. A more recent and independent study confirmed that PfPP1 is essential for blood stage parasite survival [7].

\section{PP1 holoenzymes as drug targets}

In eukaryotic cells PP1 and PP2A together are responsible for more than $90 \%$ of the protein phosphatase activity. However, in terms of substrate diversity, PP1 is predicted to catalyze the majority of protein dephosphorylation events; it is estimated that around a third of all eukaryotic proteins are dephosphorylated by PP1. PP1 has been studied since the 1940s and identified as the enzyme responsible for the conversion of phosphorylase A to phosphorylase $\mathrm{B}$. This finding, together with the discovery of phosphorylase kinase, marked the beginning of an era for the study of protein phosphorylation/dephosphorylation as a regulatory mechanism. The capacity of PP1 to dephosphorylate a considerable number of substrates was subsequently clarified by its capacity to interact with diverse binding partners/ regulators. The nature of the partner can precisely target the holoenzyme to a specific subcellular compartment and inhibit its activity or serve as a substrate. The PP1 catalytic subunit (PP1c) is an approximately $37-\mathrm{kD}$ protein and is highly conserved throughout eukaryotes. In mammals, PP1c is encoded by three different genes each undergoing alternative splicing, however, in Plasmodium only one isoform of PP1c has been described. Concerning regulatory subunits, these are multiple - more than 150 interacting proteins have been described in mammals - and structurally quite different. Consequently, although PP1c may not be a selective drug target, targeting binding partners and/or their binding sites to PP1 is a more appropriate option for the development of new strategies aiming to block specifically the functions of PP1. The feasibility of this approach was supported in recent work where a selective inhibition of the human PP1 regulator GADD34 by guanabenz, a small molecule that dissociates the GADD34-PP1 complex, showed a proteostatic action and protection of stressed cells [8].

In Plasmodium, few PP1-conserved regulatory subunits have been reported: so far, three gene products, Pf Leucine Rich Repeat 1 (PfLRR1), Pf Inhibitor 2 (PfI2) and Pf Inhibitor 3 (PfI3) have been shown to exert regulatory functions on PfPP1 activity [9-11]. Interestingly, reverse 
genetic approaches revealed that PfI2 and PfI3 are likely essential for parasite growth, thus highlighting the suitability of targeting these regulators. In general, the majority of regulators bind PP1 via a primary PP1-binding motif, the $\mathrm{RVxF}$ motif. Apart from the RVxF motif, PP1 regulatory proteins mostly lack any apparent sequence similarity, although additional interaction sites, such as SILK, MyPhoNE, FxxR/ $\mathrm{KxR} / \mathrm{K}$ and $\Phi \Phi$ motifs have been described. PfI 2 and PfI 3 exhibit the short RVxF binding motif, and mutational studies together with NMR analysis showed that it is the main binding motif to PfPP1, but also revealed that PfI2 contains a second binding motif following the $\mathrm{FxxR} / \mathrm{KxR} / \mathrm{K}$ consensus, and acting in concert with the RVxF motif to bind PP1 [12]. Based on these observations, we assumed that interfering with PfPP1 holoenzymes may create new ways for developing antimalarial compounds. We used competing peptides derived from $\mathrm{RVxF}$ and FxxR/KxR/K motifs of PfI2 and PfI3, fused to a cationic penetrating sequence and showed that these peptides not only disrupt the interaction between PP1 and its regulators, but also inhibit $P$. falciparum growth in vitro. These results provide a proof of concept and demonstrate that the interface between PP1 and its regulatory subunits may be a relevant target for the design of new antimalarials.

\section{Future directions}

In Plasmodium, we have demonstrated that peptides competing with the interaction motifs between PP1 and some of its conserved regulators inhibit parasite growth in vitro. However, targeting PP1-regulator interactions that are conserved in Plasmodium and its human host does not seem highly attractive due to the risk of side effects. Consequently, it is crucial to take advantage of the sequence differences (deletions, mutations) to design selective inhibitors with minimal unwanted effects. In parallel and in order to obtain a more selective effect on the functions of PfPP1, it is of interest to identify unique and essential PP1 regulators expressed by the parasite. This could be achieved using experimental approaches, such as $\mathrm{Y} 2 \mathrm{H}$ screening using PfPP1 as bait, and/ or in silico approaches such as virtual screening. This latter strategy has been used very recently to identify novel small inhibitors of the PfAtg8-Atg3 protein-protein interaction and the authors have shown that, together with structure-aided design, it allowed the identification of new hits for drug development [13]. It is worth noting that targeting therapeutically relevant protein-protein interactions with peptides or small molecules as antiparasitic drugs is an emerging and promising strategy [14]. Peptides have high potency and low toxicity, and various modifications can be applied to overcome the limitations due to poor stability and inefficiency in crossing cell membranes. Cyclization, $\mathrm{N}$-terminal or C-terminal modifications or alteration of the amine bond were shown to improve stability, bioavailability and selectivity.

Another critical point that would help in defining relevant hits resides in developing structural biology approaches. A crystal structure of PfPP1 with a given regulatory subunit would constitute a starting point for further selective and potent virtual screening. To date only three Pf phosphatases crystal structures have been solved and two can be considered valid active phosphatases [15-17]. More generally, this strategy could be beneficially applied to other Plasmodium phosphatases, especially those that have been recently described as possibly essential and/or those specific to the parasite [6]. Combined with biochemical studies of phosphatase activity and partner identification, we believe that this would provide leads with great potential in the discovery of new antimalarials.

\section{Acknowledgements}

The authors would like to thank the excellent work of PhD students (W Daher, A Fréville and A Vandomme) and R Pierce for his critical reading of the manuscript. This work was supported by Université de Lille, CNRS, Inserm and Institut Pasteur de Lille.

\section{Financial \& competing interests disclosure}

The authors have no relevant affiliations or financial involvement with any organization or entity with a financial interest in or financial conflict with the subject matter or materials discussed in the manuscript. This includes employment, consultancies, honoraria, stock ownership or options, expert testimony, grants or patents received or pending, or royalties.

No writing assistance was utilized in the production of this manuscript. 


\section{References}

1 Rzymski T, Mikula M, Wiklik K, Brzozka K. CDK8 kinase - an emerging target in targeted cancer therapy. Biochim. Biophys. Acta 1854(10 Pt B), 1617-1629 (2015).

2 Mohanty S, Kennedy EJ, Herberg FW et al. Structural and evolutionary divergence of cyclic nucleotide binding domains in eukaryotic pathogens: implications for drug design. Biochim. Biophys. Acta 1854(10 Pt B), 1575-1585 (2015).

3 Doerig C, Grevelding CG. Targeting kinases in Plasmodium and Schistosoma: same goals, different challenges. Biochim. Biophys. Acta 1854(10 Pt B), 1637-1643 (2015).

4 Mitcheson DF, Tobin AB, Alam MM. Applying chemical genetic tools to the study of phospho-signalling pathways in malaria parasites. Biochim. Biophys. Acta 1854(10 Pt B), 1650-1656 (2015).

5 Yokoyama D, Saito-Ito A, Asao N, Tanabe K, Yamamoto M, Matsumura T. Modulation of the growth of Plasmodium falciparum in vitro by protein serine/threonine phosphatase inhibitors. Biochem. Biophys. Res. Commun. 247(1), 18-23 (1998).

6 Guttery DS, Poulin B, Ramaprasad A et al. Genome-wide functional analysis of Plasmodium protein phosphatases reveals key regulators of parasite development and differentiation. Cell Host Microbe 16(1), 128-140 (2014).

7 Zhang M, Mishra S, Sakthivel R, Fontoura BM, Nussenzweig V. UIS2: a unique phosphatase required for the development of Plasmodium liver stages. PLoS Pathog. 12(1), e1005370 (2016).

8 Tsaytler P, Harding HP, Ron D, Bertolotti A. Selective inhibition of a regulatory subunit of protein phosphatase 1 restores proteostasis. Science 332 (6025), 91-94 (2011).

9 Daher W, Browaeys E, Pierrot C et al. Regulation of protein phosphatase type 1 and cell cycle progression by PfLRR1, a novel leucine-rich repeat protein of the human malaria parasite Plasmodium falciparum. Mol. Microbiol. 60(3), 578-590 (2006).

10 Freville A, Cailliau-Maggio K, Pierrot $\mathrm{C}$ et al. Plasmodium falciparum encodes a conserved active inhibitor-2 for protein phosphatase type 1: perspectives for novel anti-plasmodial therapy. BMC Biol. 11, 80 (2013).

11 Freville A, Landrieu I, Garcia-Gimeno MA et al. Plasmodium falciparum inhibitor-3 homolog increases protein phosphatase type 1 activity and is essential for parasitic survival. J. Biol. Chem. 287(2), 1306-1321 (2012).

12 Freville A, Tellier G, Vandomme A et al. Identification of a Plasmodium falciparum inhibitor-2 motif involved in the binding and regulation activity of protein phosphatase type 1. FEBS J. 281(19), 4519-4534 (2014).

13 Hain AU, Miller AS, Levitskaya J, Bosch J. Virtual screening and experimental validation identify novel inhibitors of the Plasmodium falciparum Atg8-Atg3 protein-protein interaction. ChemMedChem doi:10.1002/ cmdc.201500515 (2016) (Epub ahead of print).

14 Qvit N, Crapster JA. Peptides that target protein-protein interactions as an antiparasite strategy. Chimica Oggi-Chemistry Today 32(6), 31-36 (2014).

15 Bosch J, Paige MH, Vaidya AB, Bergman LW, Hol WG. Crystal structure of GAP50, the anchor of the invasion machinery in the inner membrane complex of Plasmodium falciparum. J. Struct. Biol. 178(1), 61-73 (2012).

16 Hills T, Srivastava A, Ayi K et al. Characterization of a new phosphatase from Plasmodium. Mol. Biochem. Parasitol. 179(2), 69-79 (2011).

17 Guggisberg AM, Park J, Edwards RL et al. A sugar phosphatase regulates the methylerythritol phosphate (MEP) pathway in malaria parasites. Nat. Commun. 5, 4467 (2014). 